\title{
Improved Light Extraction Efficiency by Photonic Crystal Arrays on Transparent Contact Layer Using Focused Ion Beams
}

\author{
G.M. WU ${ }^{a, *}$, B.H. TsAI ${ }^{a}$, S.F. KUnG ${ }^{b}$ AND C.F. WU ${ }^{c}$ \\ ${ }^{a}$ Institute of Electro-Optical Engineering, Chang Gung University, Kweisan, Taoyuan 333, Taiwan R.O.C. \\ ${ }^{b}$ Department of Dentistry, Chang Gung Medical Center, Kweisan, Taoyuan 333, Taiwan R.O.C. \\ ${ }^{c}$ Department of Applied Mathematics, National Dong Hwa University, Hualien 974, Taiwan R.O.C. \\ Nitride-based thin-film materials have become increasingly important for the high brightness light-emitting \\ diode applications. The improvements in light extraction and lower power consumption are highly desired. \\ Although the internal quantum efficiency of GaN-based LED has been relatively high, only a small fraction of \\ light can be extracted. In this study, a new design of two-dimensional photonic crystal array has been prepared on \\ the top transparent contact layer of indium-tin oxide film to improve the light extraction efficiency using focused \\ ion beam. The acceleration voltage of the Ga dual-beam nanotechnology system SMI 3050 was $30 \mathrm{kV}$ and the \\ ion beam current was $100 \mathrm{pA}$. The cylindrical air holes had the diameter of $150 \mathrm{~nm}$ and depth of $100 \mathrm{~nm}$. The \\ micro photoluminescence analysis results showed that the light output intensity could be 1.5 times of that of the \\ non-patterned control sample. In addition, the structural damage from the focused ion beam drilling of GaN step \\ could be eliminated. The excellent $I-V$ characteristics have been maintained, and the external light extraction \\ efficiency would be still improved for the LED devices.
}

PACS: 81.16.Rf, 42.70.Qs, 41.75.Ak

\section{Introduction}

Light-emitting diodes (LEDs) with high brightness have currently drawn much attention for a variety of applications in printers, flat panel displays, automobiles, and special lightings [1]. The improvements in light extraction and lower power consumption are highly desired [2]. Although the internal quantum efficiency (QE) of nitride-based LED has been relatively high, only a small fraction of light can be extracted, because of the interface refraction between the high index semiconductors and the ambient environment. It is thus crucial to improve the external quantum efficiency of III-nitride LEDs.

Several techniques have been proposed to improve the light extraction [3-6]. Surface roughening by wet etching could reduce internal light reflection and scatter more light outward. The uniformity has been difficult to control. A more precise control of the surface nanopatterning could be developed by high energy and excellent precision focus ion beam (FIB) technology [7]. The flexibility in pattern design is a major advantage over the other techniques. The fundamental optical characteristics can be investigated with many different periodical structures. Even some defects can be included for study. However, the incorporation of two-dimensional photonic crystal (2D-PC) air hole array could damage the brittle

* corresponding author; e-mail: wu@mail.cgu.edu.tw and fragile surface of GaN LED device by the focused ion beam. An effective design is still needed for this new era of study.

In this paper, we prepared InGaN/GaN semiconductor LED on sapphire substrates by low-pressure metal-organic chemical vapor deposition (MOCVD) with peak emission wavelength of $468 \mathrm{~nm}$. The 2D-PC air hole arrays were produced by focus ion beam SMI 3050 system. The FIB etching technique used an automatic computer program to reduce the number in process steps and no photomask would be needed. The various lattice period constants were employed for both the transparent contact layer (TCL) of indium-tin oxide (ITO) film surface and the $p$-type GaN surface of the LED devices. Bandsolve simulation tools could be used to understand the photonic band structures. The fundamental photon extraction was measured by micro photoluminescence (PL), and the light output-current $(L-I)$ characteristics were analyzed by charging with Keithley 2430 power supply. In addition, the electrical properties were revealed by Keithley 6485 picoammeter.

\section{Experimental}

The epitaxial layers of blue GaN LEDs were grown on c-plane (0001) sapphire substrates by low-pressure metal organic chemical vapor deposition (LP-MOCVD) system. During the growth, trimethylgallium (TMGa), trimethylaluminum (TMAl), trimethylindium (TMIn), and ammonia $\left(\mathrm{NH}_{3}\right)$ were used as precursors of $\mathrm{Ga}, \mathrm{Al}, \mathrm{In}$, and $\mathrm{N}$, 
respectively. The carrier gas was $\mathrm{H}_{2}$ and $\mathrm{N}_{2}$, respectively, for the growth of GaN and InGaN. At first, the growth of a $50 \mathrm{~nm}$ thick GaN buffer layer was carried out, then a $2 \mu \mathrm{m}$ undoped GaN layer and a $2 \mu \mathrm{m} n$-type GaN layer. The active multiple quantum well (MQW) layers consisted of six periods of $4 \mathrm{~nm}$ thick wells and $12 \mathrm{~nm}$ thick barriers. The final $p$-type GaN layer had a thickness of $0.18 \mu \mathrm{m}$. The designated emission peak wavelength was $468 \mathrm{~nm}$.

We fabricated 2D triangular PC air hole arrays on the top TCL of ITO on the GaN LED devices. The schematic structure is shown in Fig. 1. There were four steps to process from LED base wafer to device. The processes included MESA, TCL (Ni/ITO, 2/270 nm), n-pad (Cr/ $\mathrm{Au}, 20 / 100 \mathrm{~nm})$ and $p$-pad $(\mathrm{Cr} / \mathrm{Au}, 20 / 100 \mathrm{~nm})$. The size of the LED device was $270 \times 130 \mu \mathrm{m}^{2}$ with top and bottom pads and the lighting area was $150 \times 80 \mu \mathrm{m}^{2}$. The diameter of the air hole was $150 \mathrm{~nm}$ and the depth was $100 \mathrm{~nm}$, drilled by accelerated Ga ions. The acceleration voltage has been $30 \mathrm{kV}$ and the ion beam current was $100 \mathrm{pA}$. The cylindrical air hole arrays exhibited varied periods of 300-700 nm in this study.

\section{FIB method}

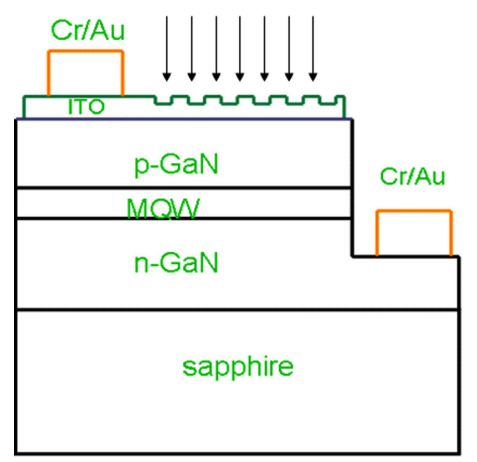

Fig. 1. The schematic diagram of the GaN LED with 2D PC array on the top transparent contact layer.

The micro photoluminescence (PL) was employed to measure the LED device optical performance using $\mathrm{He}-\mathrm{Cd}(325 \mathrm{~nm})$ laser. The current-voltage $(I-V)$ characteristics were evaluated at room temperature by a Keithley 2430 power supply, with a forward voltage from $-3 \mathrm{~V}$ to $5 \mathrm{~V}$ and the limiting current was $5 \mathrm{~mA}$. In addition, the light output versus current $(L-I)$ characteristics were studied using Keithley 6485 picoammeter and Keithley 2430 power supply. The luminescence intensity phenomena of these samples were measured as functions of the forward injection currents up to $20 \mathrm{~mA}$.

\section{Results and discussion}

Figure 2 shows the scanning electron micrographs of the triangular air hole arrays on the top transparent contact layer of ITO on the nitride LED devices. The period has been ranging from 300 to $700 \mathrm{~nm}$. The focus ion beam technology has been quite effective in creating the desired nanoscale patterns on ITO film. The nominal depth was $100 \mathrm{~nm}$ and the ITO film thickness was $270 \mathrm{~nm}$

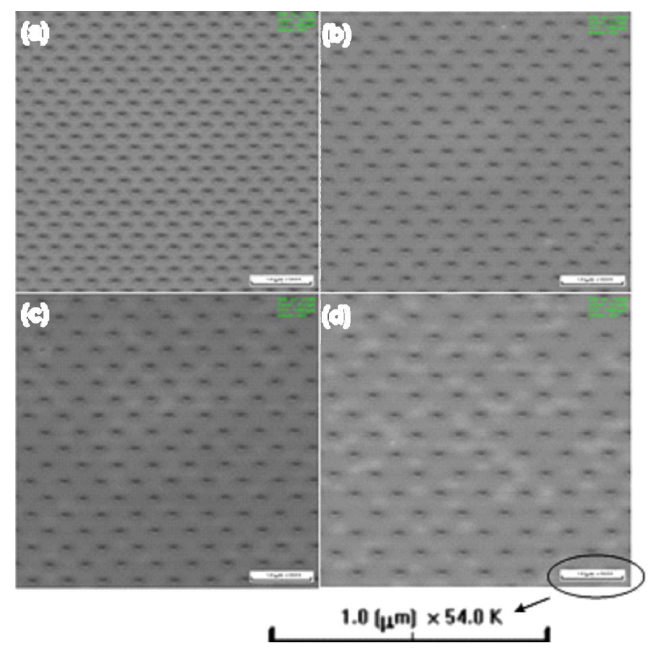

Fig. 2. Scanning electron micrographs of the triangular air hole arrays on the top TCL layer of ITO on the LED devices with periods at (a) $400 \mathrm{~nm}$, (b) $500 \mathrm{~nm}$, (c) $600 \mathrm{~nm}$, and (d) $700 \mathrm{~nm}$.

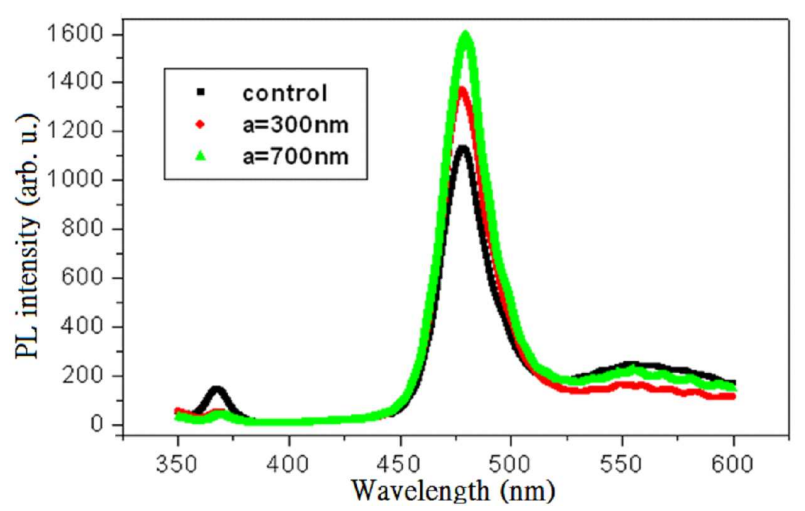

Fig. 3. The micro PL measurement results of the LED devices with the 2D-PC array period of $300 \mathrm{~nm}$ and $700 \mathrm{~nm}$, and the control sample (no 2D-PC).

The micro PL measurement results of the LED devices are shown in Fig. 3. Here, the LED samples with the $2 \mathrm{D}-\mathrm{PC}$ array period of $300 \mathrm{~nm}$ and $700 \mathrm{~nm}$, along with the control sample (no 2D-PC), are displayed. The major emission peak wavelength remained about the same. However, the micro PL data showed that the $700 \mathrm{~nm}$ period PC-LED exhibited a light output that was 1.5 times of that of the control LED sample without the photonic crystal array. The PC-LED device of the period of $300 \mathrm{~nm}$ also presented an improvement factor of 1.2 when compared with that of the control sample. The extraction efficiency was partly improved by the redirection of light from scattering, which allowed more photons to escape from the light cone. The effect of photonic crystal 
array in the emitted light wavelength range played another role.

Figure 4 provides an example of the photonic band diagram of the LED with $300 \mathrm{~nm}$ period array by the Bandsolve simulation tools. A photonic band gap was observed in the frequency range of $0.28-0.32(\omega=a / \lambda)$, where $a$ is the period of photonic crystal pattern. The photons could propagate in the corresponding modes and became prohibited in the other directions.

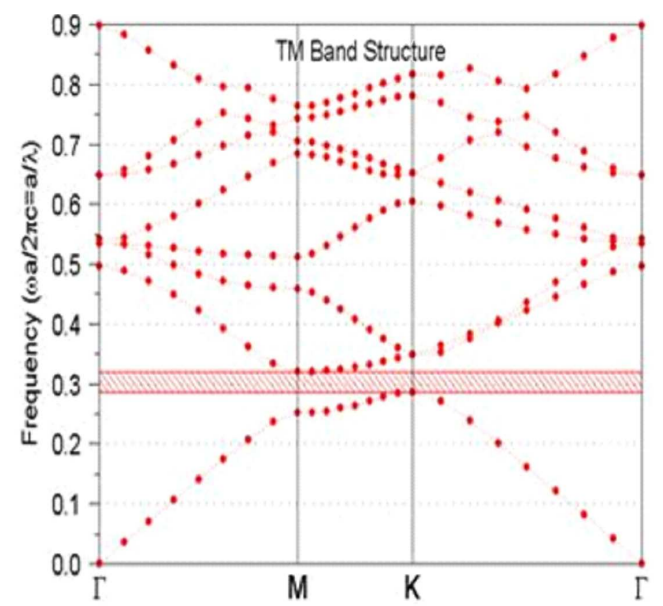

Fig. 4. Photonic band structure of the LED with $300 \mathrm{~nm}$ period array by the Bandsolve simulation tools.

The light output-current $(L-I)$ characteristic curves of the LED device samples with different 2D-PC pattern periods are shown in Fig. 5. The injection current has been raised from 0 up to $20 \mathrm{~mA}$. A much higher injection current was avoided, because it could damage such a small device. The light output intensity exhibited 10-22\% improvement over that of the control sample without patterning.

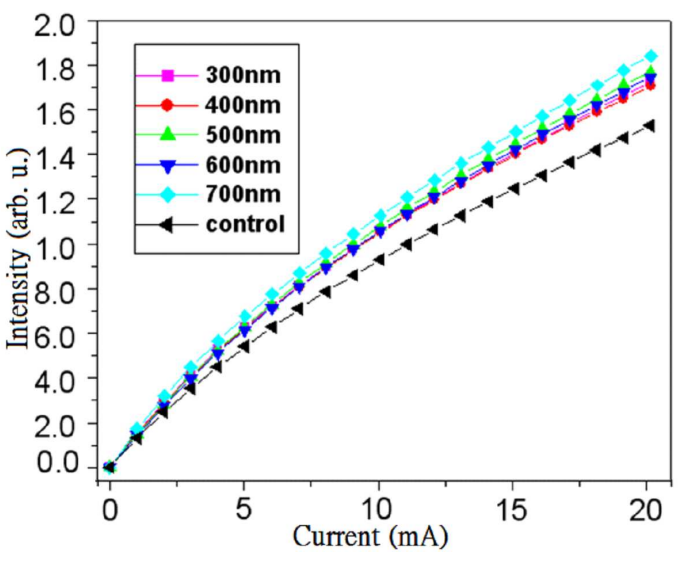

Fig. 5. The light output-current $(L-I)$ characteristic curves of the LED device samples with different 2D-PC pattern periods on TCL. The control sample had no patterning.

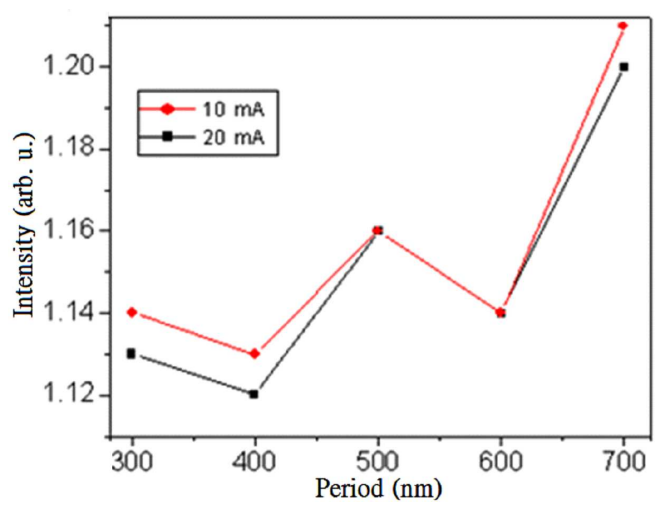

Fig. 6. The light output intensity data of the LED devices at the two injection current levels, $10 \mathrm{~mA}$ and $20 \mathrm{~mA}$.

Figure 6 summarizes the light output intensity data of the LED devices at the two injection current levels, $10 \mathrm{~mA}$ and $20 \mathrm{~mA}$. The constructive interference from the air hole array has contributed to the enhancement of light extraction [8]. The $700 \mathrm{~nm}$ period LED sample showed the most significant improvement. The trend was in agreement with that of the corresponding micro PL results. The current-voltage $(I-V)$ characteristics were measured at room temperature to reveal the situation in power consumption.

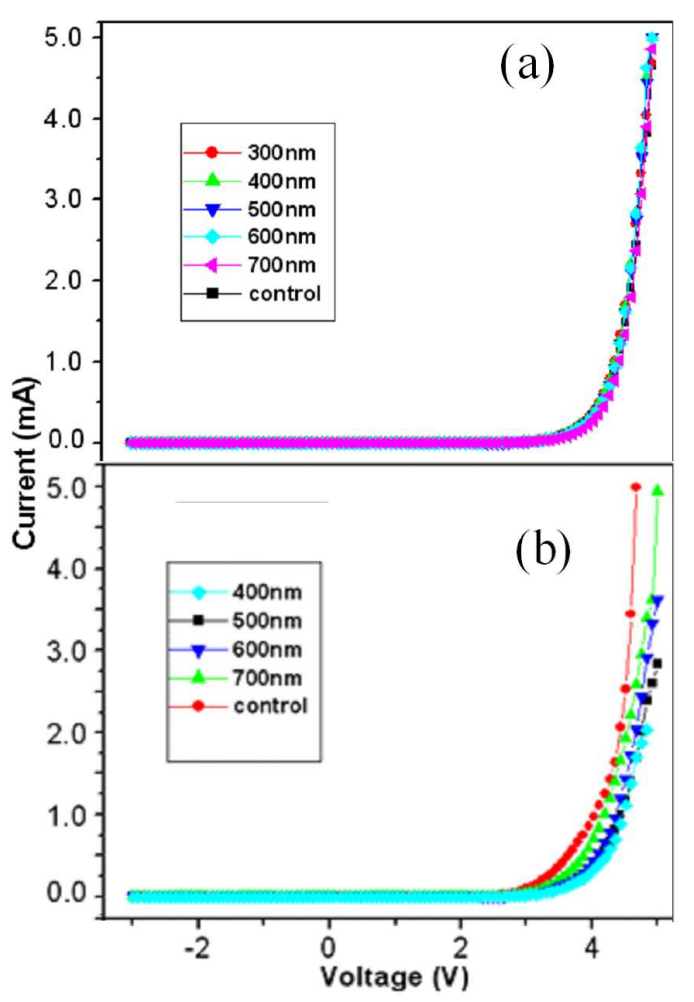

Fig. 7. The current-voltage $(I-V)$ characteristic curves for the LED devices measured at room temperature: (a) 2D-PC array on top TCL layer of ITO film, and (b) 2D-PC array on $p$-GaN layer. 
The $I-V$ characteristic curves of the PC-LED samples are shown in Fig. 7. The operating current has been pretty much the same at the forward voltage from $-3 \mathrm{~V}$ to $5 \mathrm{~V}$ for both the PC-LED device samples and the control sample. In addition, the $I-V$ characteristic curves of the PC- $p$-GaN-LED samples are also included in the figure for the purpose of comparison. These PC- $p$-GaN-LED samples had their photonic crystal air hole array patterned on the $p$-GaN layer, before the transparent contact layer of ITO film was coated (also see Fig. 1).

It could be noted in general that the operating current was reduced for the forward voltage $3-5 \mathrm{~V}$, suggesting an increase in electrical resistance. This was attributed to the incorporation of the triangular photonic crystal array on the $p$-GaN layer of the LED device samples. The operation voltage was also increased from $4.19 \mathrm{~V}$ to $4.51 \mathrm{~V}$ at the same operating current of $1 \mathrm{~mA}$ when compared with the control sample. It was likely caused by the structural damage from the FIB processing of the air hole array on the $p$-GaN layer. The Ga ion beam was very much focused and had high acceleration voltage. It could create high stress and distortion when bombarding a thin film layer. The ohmic contact area was thus reduced.

On the other hand, the photonic crystal array on the ITO surface film did not seem to have increased the operation voltage. The possible damage could not be discernible. This was due to the better heat and stress distribution during the FIB processing on the top ITO film. Therefore, the new photonic crystal air hole array design on the top ITO film surface has been demonstrated to be effective in the improvement of external light extraction without affecting the $I-V$ characteristics of the LED devices. The energy efficiency would be increased and the power consumption could be improved.

\section{Conclusion}

In this study, we designed and fabricated triangular photonic crystal arrays on the top ITO film surface of GaN LED devices. It has been successfully demonstrated to improve the external quantum efficiency by using the new two-dimensional photonic crystal air hole array. The nanoholes were produced by focused ion beam method using accelerated $\mathrm{Ga}$ ions. The light extraction became dependent on the pattern period. The most constructive interference was found for the $700 \mathrm{~nm}$ period sample. From the $I-V$ measurement results, it was noted that the operation voltage could be increased from $4.19 \mathrm{~V}$ to $4.51 \mathrm{~V}$ with the photonic crystal array processed on the $p$-GaN layer. This was attributed to the structural damage from the FIB drilling GaN step. The ohmic contact area was reduced, and the resistance became increased. The problem was solved by incorporating the photonic crystal array on the top transparent contact layer of ITO film surface. The excellent $I-V$ characteristics were preserved, while the external light extraction efficiency would be still improved for the LED devices.

\section{Acknowledgments}

This work was partially supported by the National Science Council under research grant NSC98-2221-E182-001 .

\section{References}

[1] J. Shakya, K.H. Kim, J.Y. Lin, H.X. Jiang, Appl. Phys. Lett. 85, 142 (2004).

[2] D.H. Kim, C.O. Cho, Y.G. Roh, Appl. Phys. Lett. 87, 203508 (2005).

[3] Y.S. Lin, K.J. Ma, C. Hsu, S.W. Feng, Y.C. Cheng, C.C. Liao, Appl. Phys. Lett. 77, 19 (2000).

[4] T. Fujii, Y. Gao, R. Sharma, E.L. Hu, S.P. DenBaars, S. Nakamura, Appl. Phys. Lett. 84, 855 (2004).

[5] H. Kim, J. Cho, J.W. Lee, S. Yoon, H. Kim, C. Sone, Y. Park, T.Y. Seong, Appl. Phys. Lett. 90, 161110 (2007).

[6] G.M. Wu, Z.D. You, Solid State Electron. 51, 1351 (2007).

[7] G.M. Wu, Z.J. Cai, J.C. Wang, T.E. Nee, Surf. Coat. Technol. 203, 2674 (2009).

[8] D.H. Long, I.K. Hwang, S.W. Ryu, Jpn. J. Appl. Phys. 47, 4527 (2008). 